\title{
DESDE DÓNDE NOS HABLAN LOS ESTUDIOS SOCIOCULTURALES EN CUBA
}

\section{FROM WHERE DO THE SOCIAL STUDIES IN CUBA, SPEAK US?}

\author{
Susell Gómez González*
}

\author{
RESUMEN
}

Este artículo constituye un acercamiento al protagonismo del sistema de educación cubano, con la apertura de una nueva carrera en el campo de las Ciencias Sociales, la cual demuestra las reflexiones del contexto posmoderno en la actualidad. El presente trabajo se desarrolla desde el espacio de la recreación del discurso culturológico, en el cual se trabaja su surgimiento en Cuba y sus retos, recreando algunas cuestiones, a través de pautas tales como: Se abre un nuevo camino en las ciencias sociales cubanas y La Universidad de Granma, escenario de la actuación del profesional de los Estudios Socioculturales en Cuba; en las cuales narran encuentros y desencuentros de esta nueva carrera y disciplina en la formación de su profesional.

PALABRAS CLAVE: CUBA * EDUCACIÓN *CIENCIAS SOCIALES * CULTURA * SOCIEDAD * METODOLOGÍA

\section{ABSTRACT}

This article presents an approach to the role of the Cuban education system, with the opening of a new career in the field of Social Sciences, which shows the reflections of the postmodern context today. This is developed from the recreation room culturologic speech, even working its appearance in Cuba and its challenges, recreating some guidelines, such as: Opens a new path in Cuban social sciences and the University of Granma, the scene of professional performance of Sociocultural Studies in Cuba, which narrate encounters and misunderstandings of this new career and discipline in their professional training.

KEYWORDS: CUBA * EDUCATION * SOCIAL SCIENCES * CULTURE * SOCIETY * METHODOLOGY

Facultad de Ciencias Sociales y Humanísticas de la

Universidad de Granma (Cuba).

sgomezg@udg.co.cu 


\section{NUEVO CAMINO EN LAS CIENCIAS SOCIALES CUBANAS}

El análisis de los contextos sociales y los campos culturales limitan a un espacio marcadamente sociológico o artístico, en este sentido, se hace pertinente la mirada desde lo complejo $y$ diverso que se vuelve cada vez más el espacio cultural, social, político, económico y ambiental en el que se desarrolla el hombre.

Ganando protagonismo en este sentido, en el sistema educacional cubano, de acuerdo con el Ministerio de Cultura, se abrió una nueva carrera universitaria: Licenciatura en Estudios Socioculturales, en el curso 1998-1999 con carácter experimental, en la Universidad de Cienfuegos y a partir del curso 1999-2000, se da en otras universidades del territorio nacional.

Esta carrera parte de un perfil amplio en el campo de las ciencias sociales, centrando el objeto de trabajo del egresado de la carrera, en los procesos culturales que ocurren en diferentes contextos sociales, especialmente en aquellos que inciden en el incremento de la calidad de la vida colectiva, el enriquecimiento espiritual, el fortalecimiento de la identidad cultural y la capacidad de participación de la población en dichos procesos.

Los campos de acción de la carrera son: la gestión y la promoción sociocultural, la teoría y la metodología social, la historia y el pensamiento cultural y la cultura cubana ${ }^{1}$.

Se espera que este profesional pueda actuar en la identificación y el desarrollo del potencial cultural de los territorios cubanos, la investigación, la programación y la gestión de proyectos sociales, el trabajo sociocultural comunitario, así como, la docencia y la extensión cultural que se realiza desde instituciones, organizaciones, comunidades, empresas $y$ otras entidades. Actualmente, los egresados de la carrera pueden trabajar en instituciones culturales y educativas del sistema nacional de enseñanza y del subsistema de la enseñanza artística.

Para la misma se han diseñado modos de actuación, los cuales implican habilidades para la detección, investigación e intervención socio- cultural. De acuerdo a lo que se ha planteado por su comisión nacional de carrera, existen una serie de objetivos generales para la misma en los que se encuentran:

$\diamond \quad$ Revelar en su accionar cotidiano su compromiso político ideológico para participar en la construcción del proyecto social socialista cubano $y$ de otros proyectos sociales que tengan al ser humano y su calidad de vida como centro.

$\diamond \quad$ Desarrollar científicamente, sobre la base de una concepción de mundo materialista dialéctica y de una ética socialista, sus complejas tareas profesionales en la solución de los problemas que la construcción del Socialismo plantea en la esfera sociocultural y que se concretan de forma específica en sus campos de acción dados.

$\diamond \quad$ Demostrar niveles de dominio integral de conocimientos $y$ herramientas científico-metodológicas que les permitan consolidar continuamente una visión histórico-lógica del desarrollo social, de la praxis cultural de la sociedad y consecuentemente, contribuir al incremento y consolidación de la participación de la población y protagonismo de los diferentes sujetos sociales individuales y colectivos implicados en este proceso.

$\diamond \quad$ Desarrollar de forma permanente $y$ con independencia procesos de superación profesional que incluyan aspectos: político ideológicos, científicos, técnicos, culturales, físicos, así como, las que se relacionan con las crecientes formas de uso y aprovechamiento de las nuevas tecnologías de la información y las comunicaciones, todos necesarios y pertinentes a su desarrollo personal y a su incidencia colectiva en el orden profesional.

$\diamond \quad$ Gestionar desde diferentes roles (directivos, asesores, evaluadores y/o participantes), investigaciones socioculturales, programas y proyectos de promoción y animación sociocultural, acciones de extensión cultural, formación y capacitación docente que contribuyan al enrique-

1 Documentos metodológicos de la carrera. 
cimiento de la participación protagónica de la población en su propio desarrollo sociocultural.

$\diamond \quad$ Utilizar de forma pertinente $y$ adecuada, como recurso de obtención de información, comunicación y acción profesional la lengua materna, tanto escrita como oral, el inglés como lengua extranjera $y$ las nuevas tecnologías de la informática $y$ las comunicaciones.

$\diamond \quad$ Asumir la realización sistemática de la actividad deportiva y la recreación como recursos de crecimiento social y personal. Lograr la preparación necesaria para, como profesional y como ciudadano, asumir actitudes $y$ desplegar habilidades en la defensa, tanto en su sentido general como en lo relativo a la defensa civil ${ }^{2}$.

Así también, la carrera a nivel nacional, ha diseñado un conjunto de habilidades profesionales que debe poseer el egresado de la misma, entre estas están:

$\diamond \quad$ Desarrollar adecuadamente la actividad de animación, gestión y promoción sociocultural en los diferentes contextos de su posible ubicación laboral. Identificar $y$ actuar en correspondencia con el desarrollo del potencial cultural del entorno social en que se desenvuelve profesionalmente, contribuyendo con sus acciones al incremento de la riqueza cultural del territorio, privilegiando la participación y la promoción de artistas, intelectuales, creadores y portadores de tradiciones, así como, otros técnicos y profesionales, como son promotores culturales, instructores de arte, trabajadores sociales y líderes comunitarios, entre otros.

$\diamond \quad$ Participar activamente en la programación y gestión de proyectos sociales, contribuyendo a la utilización de los diferentes saberes con enfoque transdisciplinar $y$ contribuir con ello a la

2 Documentos rectores de la Carrera Nacional de Estudios Socioculturales. Ministerio de Educación Superior, Cuba. solución de los problemas que la construcción del Socialismo plantea en la esfera sociocultural $y$ que se concretan de forma específica en sus campos de acción dados. Diseñar y gestionar acciones de extensión cultural a realizar desde instituciones, organizaciones, comunidades, empresas y otras entidades. Asumir acciones de docencia y capacitación en el campo de su perfil profesional. Realizar una adecuada actividad científico-investigativa en el área sociocultural como parte de su contenido de trabajo.

$\diamond \quad$ Adecuar sus acciones profesionales a la realidad sociocultural de los espacios donde incida, ya sea zonas urbanas o rurales, incluidas aquellas de difícil acceso o determinadas por especificidades en dicho territorio de distintos grupos étnicos, generacionales o de género, siempre desde el respeto a la diversidad.

$\diamond \quad$ Propiciar procesos de concertación y/o coordinación entre agentes sociales como son las instancias del gobierno, instituciones culturales, educacionales, organizaciones políticas $y$ de masas y otras entidades de interés interactuantes en el entorno de su actividad profesional.

$\diamond \quad$ Asesorar a los diferentes agentes sociales presentes en los procesos socioculturales en los que participa, los cuales requieran de sus servicios profesionales.

$\diamond \quad$ Asumir el compromiso político ideológico de participación en la construcción del proyecto social socialista cubano y de otros proyectos sociales que tengan al ser humano y su calidad de vida como centro. Propiciar la participación y el protagonismo de los diversos sujetos sociales implicados en el proceso del desarrollo sociocultural del territorio en que realiza su actividad profesional, referenciando el enriquecimiento espiritual y cultural de la sociedad, en correspondencia con el proyecto social vigente.

$\diamond \quad$ Concebir $y$ ejecutar acciones de superación y capacitación autogestionadas que le permitan lograr un proceso permanente y autónomo de apropiación de los 
recursos profesionales necesarios $y$ pertinentes a su desarrollo personal y a su incidencia, en el orden humano y laboral, al del resto de los técnicos y las personas con los que interactúa cotidianamente. Utilizar de forma pertinente $y$ adecuada, tanto en su forma oral como escrita, la lengua materna como recurso de obtención de información, comunicación y acción profesional.

$\diamond \quad$ Utilizar adecuadamente el idioma inglés como lengua extranjera, así como, las nuevas tecnologías de la informática y las comunicaciones en el ejercicio de la profesión y en su desarrollo personal. Asumir actitudes y desplegar acciones comprometidas con la defensa del país y la cultura nacional, tanto en su sentido general como en lo relativo a la defensa civil, especialmente con una atención privilegiada a los recursos socioculturales relacionados con las mismas ${ }^{3}$.

A partir de lo antes expuesto, se puede decir que en Cuba se ha abierto una brecha interesante, en cuanto a la formación de un profesional de perfil amplio que propiciará un cambio en la realidad sociocultural del país.

Esto ha sido objeto de análisis y discusión en sesiones académicas, manifestándose varias posiciones tanto a favor como en contra, ya que resulta una experiencia novedosa a la que le tomará tiempo ganarse el espacio y el reconocimiento, constituyéndose una preocupación el trabajo profesional, pues el organismo empleador es el Ministerio de Cultura mayoritariamente y resulta una contradicción, que a pesar de que los egresados están preparados para asumir la mayoría de las acciones en instituciones, se evidencia resistencia a dejarlos entrar y falta de confianza en su preparación.

Si bien es cierto lo antes mencionado, a partir de la importancia que reviste esta nueva disciplina en el ámbito del pensamiento cultural cubano, se debe enfatizar en la construcción epistémica que da origen a la misma y se debe trazar una pregunta que guie este trabajo:

3 Modelo del profesional de Estudios Socioculturales.
¿Resulta necesario en el contexto cubano la formación profesional de esta carrera en las ciencias sociales?

La historia de esta disciplina en Cuba, dejará entre ver por si sola, la necesidad no solo en el contexto cubano sino en el latinoamericano, pues la complejidad y el entramando de mediaciones culturales, políticas y sociales, hacen de la realidad un campo pluridimensional y antagónico, para el que se requiere un análisis desde un estudio sociocultural que transversalise la propia dinámica social y el comportamiento cultural humano.

¿LO SOCIOCULTURAL, UNA NUEVA PERSPECTIVA DE ANÁLISIS EN EL CONTEXTO CULTURAL CUBANO?

Para comenzar este recuento, se hace imprescindible delimitar qué entender por sociocultural y a qué remite este término. Muchas han sido las formas de explicación del mismo, desde criterios en oposición de detractores de la carrera en el proceso cubano. Lo primero que asumen los profesionales de las ciencias sociales en Cuba, es que esta carrera se constituye una idea no fundamentada desde el pensamiento científico o también surgen ideas tales como: para qué esta carrera si un Lic. en Sociología puede hacer el trabajo de trasformación social o un Lic. en Filología puede estudiar la cultura cubana y sus procesos actuales. El interés de la autora no es defender a ultranza una nueva área del saber partiendo de posiciones de colegas de esas ciencias antes mencionadas, sino reivindicar el conocimiento $y$ las potencialidades de la carrera y entiéndase de lo sociocultural para explicar la realidad sociocultural, partiendo de este mismo término ¿qué se entiende entonces por sociocultural?

La última centuria; sin embargo, ha obligado al cuestionamiento de los márgenes disciplinarios, además en el necesario mirar hacia las raíces, se han encontrado áreas no esclarecidas en el orden epistemológico, pues los intereses de indagación que las realidades sociales constituían como encargo para ambas ciencias lo determinaron así y también es válido añadir que la complejidad del asunto sugería mirar hacia otro lado. La lógica interna evolutiva de 
ambas disciplinas científicas no estaba lista para ello, desposeídas de herramientas cognitivas e instrumentales para explicar el por qué y el cómo; se podía constatar la inexistencia de uniformidad entre los estudiosos en la utilización de los conceptos "sociedad" y "cultura". Para muchos investigadores de estas áreas de saber — según Kroeber y Parsons ${ }^{4}$ — es innecesaria la distinción entre sociedad y cultura, ya que toda la esfera de la vida social de las personas es sociocultural.

Es necesario señalar el vacío teórico en este sentido, han sido pocos los artículos que referencian este término como antecedente, pero se puede consultar la monografía ¿Qué son los estudios socioculturales? de Rafael Freyre, pues existe una tendencia a establecer este término como apellido a disímiles cuestiones, pero pocos han sido los que les ha interesado establecer finalmente qué es y a qué remite, partiendo del supuesto de que en un inicio se adolecía de una visión de esta, hoy día luego de más de seis años de graduaciones de egresados en este campo y de experiencias desde la práctica cotidiana, se pueden establecer pautas de avance para la carrera al mismo tiempo que se ha ido realizando un inacabado concepto, pero existen nuevas concepciones o ideas, en fin, un conjunto de formas. En este caso en particular, se tomará como base el concepto dado por la M.Sc. Yanet Rosabal Navarro:

... un constructo postmoderno que refiere el sistema de relaciones $y$ mediaciones entre sociedad y cultura, de cuya compleja interacción resultan objetos, elementos $y$ procesos que contienen unívocamente lo social y lo cultural, de manera que resulta imposible e innecesario, establecer en su análisis una distinción ${ }^{5}$.

Estos autores realizaron un interesante y sugestivo análisis de la correlación entre los conceptos sociedad y cultura en los marcos del pensamiento sociológico y antropológico norteamericano. Citado por John Dumoulin en Cultura, Sociedad y Desarrollo. La Habana. Editorial Ciencias Sociales, 1973: 191202.

5 Rosabal Navarro, Yanet. Lo sociocultural o la recreación del discurso culturológico en los marcos del contexto científico y tecnológico post-
Para llegar a esta posición fue necesario un análisis desde las ciencias explicativas de la cultura y de la reubicación del sujeto social desde el contexto de la posmodernidad, aún cuando no es el objetivo del presente artículo hacer un análisis posmoderno del término, resulta imprescindible una explicación desde el cambio que se ha producido, con enfoques $y$ perspectivas de análisis que tradicionalmente se han empleado y que han ocupado un lugar privilegiado en la dicotomía cultura-sociedad.

Para dicho planteamiento se debe tener en cuenta el replanteamiento y la re-definición de sus objetos de estudio, la re-ubicación de los márgenes disciplinarios, la re-elaboración de los instrumentales empleados para indagar sobre la realidad social; así como, los enfoques y las perspectivas de análisis tradicionalmente utilizados.

Es por ello que para dar fin a esta dicotomía entre el pensamiento cultural y el social, se explica una coincidencia en la visualización de la cultura como fenómeno que contiene por una parte, toda reflexión sobre lo "social" y por otra, a aceptar la dimensión simbólica - lo "cultural"- como un constituyente ontológico de los procesos sociales.

Entonces las ciencias explicativas de la cultura - entiéndase por estas a la historia, la filosofía, la sociología, la antropología y la psicología, entre otras - han dado cuenta de los procesos y los fenómenos, pero diferenciados en lo social y lo cultural.

Esta limitación, desde una posición social y cultural, es lo que se entenderá por la limitación de estas ciencias para explicar una realidad que manifiesta unívocamente estas dos concepciones, pues se ha parcializado el abordaje transdisciplinar en el estudio de la realidad que en este momento aún no se le pondrá sociocultural, pero que al ser permeada de las interacciones del sujeto social y culturalmente formado en su actuar cotidiano, le ha hecho mucho daño a la explicación del proceso aún social y cultural.

Se considera que al ser el contexto postmoderno el máximo responsable del fin de la demarcación y diferenciación entre sociedad y cultura, se ha logrado complejizar el panorama

moderno. Pág. 15. 
de estas ciencias en la formación universitaria cubana. Se habla del fin de la historia, de la imposibilidad de construir sistemas filosóficos generales, de la caducidad de los paradigmas de investigación sociológica para la comprensión de la nueva sociedad, de la inexistencia de lo exótico y con ello la pérdida del objeto de estudio antropológico, de la disolución del sujeto, de la re-invención de la economía, etc. Todos estos "cuestionamientos" son más que expresiones de la crisis al interior de las teorías científicas que explicitan la acción humana, resultado del desconcierto ante una situación novedosa $y$ diferente que remueve los cimientos de una etapa anterior - la Modernidad - y que obliga a un nuevo "saber hacer", pues nuevas son las preguntas y las respuestas a buscar.

Se propone la idea de que el hombre es un ser humano social y cultural en el mismo momento y a la misma hora, pues hasta cuándo estará el discurso epistémico preguntándose ¿qué es lo social y qué es lo cultural? Con la aparición de esta nueva área del saber y perspectiva de análisis en las ciencias sociales cubanas, se abre una nueva forma de explicación de la realidad y dado el análisis, esta va a ser sociocultural, así como, también el hombre será entendido como un ser eminentemente sociocultural y su producción será igualmente de esta forma; todo análisis que parte del hombre deberá ser sociocultural, entendiéndosele a la realidad también sociocultural, ya que parte del resultado de la actividad cotidiana de este y de su formación como objeto de estudio desde el análisis de la realidad.

Los Estudios Socioculturales son una tipicidad postmoderna en muchos casos, hasta el punto en que han sido vistos como los únicos que dan cuenta de este momento histórico ${ }^{6}$, en las ciencias sociales y humanísticas.

Es necesario aclarar que lo sociocultural apunta a una visión de sistema que soluciona, en buena medida, las insuficiencias anteriormente abordadas. La necesidad de un concepto como este es indiscutible, ya que antes de definir

$6 \quad$ Para documentarse en esta afirmación, se puede consultar el artículo de Roberto Follari: Estudios sobre postmodernidad y estudios culturales: ¿sinónimos? Disponible en <http://www.educ.ar> cualquier característica de la vida social de los hombres y aislar los diferentes elementos que la conforman, es necesario distinguir el portador real de esta vida, es decir, el sistema que proporciona el marco de realización de la acción colectiva humana. La vida social de los hombres posee grandes dificultades para deslindarse, pues en lo fáctico, cada acción humana tiene una lectura social y cultural simultáneamente, por ende, sociocultural.

Los Estudios Socioculturales en Cuba, como formación académica, surgen con un carácter privilegiado inclinándose por la investigación sociocultural como objeto de trabajo de este profesional, pues su primer reto está en la comprensión de la realidad sociocultural, su decodificación y explicación - a partir de un proceso investigativo - para con ello, determinar los problemas a analizar y solucionar, desde el trabajo sociocultural comunitario.

La apertura de la carrera de Licenciatura en Estudios Socioculturales es sin dudas un reto, pues formar un profesional con el objetivo de que ejerza en un campo cultural complejo y en el que aún su objeto de estudio está en incipiente construcción, es un gran riesgo.

Un análisis desde los documentos normativos de la carrera, permitió dar cuenta que en ninguno se especifica que es lo sociocultural. Incluso aún cuándo se hace mención a teoría sociocultural y metodología sociocultural como campos de acción que debe dominar este profesional, los contenidos de estos campos no aparecen explicitados.

La fundamentación de la carrera toma como referentes la relevancia del ámbito comunitario en las últimas décadas de la vida sociopolítica nacional, siendo esto lógico, ya que los procesos descentralizadores de los dos últimos decenios cubanos, aunque comedidos, contribuyeron al protagonismo de los espacios locales - comunidades - en el discurso científico y político de investigadores y líderes nacionales. Al mismo tiempo, este proceso encontraba homologaciones con lo que en el mundo, paralelo al proceso globalizador, está ocurriendo: una revalorización del espacio local como determinante de los procesos macrosociales, fundamentalmente en términos de desarrollo. 
Si bien es cierto que se hace necesario el trabajo desde las comunidades, debe primarse también la investigación culturológica, teniendo en cuenta las potencialidades del campo cultural cubano y la realidad en las instituciones y centros de promoción cultural en Cuba, que parten de visiones limitadas y se promulgan en función de investigaciones descontextualizadas, obviando los referentes culturales que condicionan la propia historia de las localidades y zonas.

Existe una visión pesimista del encargo social de esta, se estima que en la actualidad esta visión persiste, lo cual está dada por lo antes dicho y por el desconocimiento de los logros investigativos que han logrado los egresados de la misma, aunque será valido señalar el cambio que se ha logrado en cuanto al pensamiento en este orden, por lo cual se ha asumido esta perspectiva de análisis para explicar de forma transdiciplinar los procesos socioculturales. Quizás en un inicio existía el temor de la no necesidad del resto de las disciplinas existentes en las ciencias sociales cubanas, dada la creación de un profesional que desde su currículo recibe una fuerte carga de disciplinas, tales como: Historia y Cultura, que recoge entre otras asignaturas tales como: Cultura Latinoamericana y del Caribe, Arte Cubano, Historia Social de la Literatura y el Arte, Apreciación del arte, Apreciación literaria, Literatura universal, Literatura cubana, Cultura cubana, Teatro cubano, Música cubana entre otras, disciplinas también como: Teoría y Metodología de la investigación social con un compendio de asignaturas como: Metodología de la investigación, Estudios de comunidades, Sociología de la cultura, Antropología cultural, Demografía, Sociología agraria y rural, etc.

También en otro orden de disciplinas se estudian una amplia gama, dándole al profesional una formación eminentemente transdiciplinar, haciéndolo capaz de realizar investigaciones en las ciencias sociales en Cuba, desde análisis literarios y tesis de orden teórico del pensamiento sociocultural, incluyendo proyectos socioculturales de trasformación, evaluaciones de impacto, procesos de sistematización de experiencias e incluso, trabajos investigativos donde se aborden elementos de la sociedad que no le atañen a otras ciencias, tal es el caso de la promoción y animación socioculturales, así como, también la programación y propuesta de trasformación a nivel institucional y comunitario. Como resultado de estas investigaciones, se han dado explicaciones $y$ comprensiones desde la vinculación paradigmática de las ciencias sociales en Cuba, que en ninguna otra carrera se había logrado hasta el momento.

Desde el mismo nombre de la carrera, se invita a pensar en un paso superior de la explicación de nuestras prácticas pues, llama la atención al ser la única carrera en Cuba en la cual su nombre indique esta necesidad de investigación sociocultural. Evidentemente, denota una etapa superior en el reordenamiento de las demarcaciones entre lo social y lo cultural, asumiendo de una vez como vanguardia en la educación superior cubana.

Se ha expuesto el criterio de la complejidad de lo sociocultural, un pensamiento que le es inherente a los estudios socioculturales, por ello la creación y apertura de la carrera debió nutrirse de este pensamiento y la idea de collage no es funcional ni pertinente en este caso, en cambio la visión holística, de sistema, articuladora de esferas e integradora de acercamientos disciplinarios, le es mucho más propicia.

Asumir el reto que representa formar un profesional para ejercer en un área de saber eminentemente transdisciplinar, puede hablar de limitaciones, de nacimientos lastrados por una ambiciosa pretensión; sin embargo, se defiende el criterio de que este profesional, desde las herramientas teórico-metodológicas que posee - aún cuando deben ser perfeccionadas-, estará en condiciones de realizar acercamientos mucho más coherentes con la realidad sociocultural de los que podrá realizar otro profesional de las ciencias sociales y humanísticas y desde el prisma de lo sociocultural; además, como perspectiva de análisis, podrá coordinar equipos multidisciplinarios que — con la transdisciplina como herramienta- logren una adecuada comprensión y explicación de los procesos socioculturales $y$ un efectivo diseño de estrategias, proyectos y programas de desarrollo, tanto a nivel local como nacional. 
LA UNIVERSIDAD DE GRANMA, ESCENARIO DE LA ACTUACIÓN DEL PROFESIONAL DE LOS ESTUDIOS SOCIOCULTURALES EN CUBA

En el año 2001, la Universidad de Granma abre sus puertas al primer grupo de estudiantes matriculados en esta carrera en la provincia, imprimiéndole con ello un sello humanístico a la misma, la cual hasta el momento había sido una universidad agraria.

En sus inicios, como ya se explicó anteriormente, esta apertura trajo consigo un proceso de validación desde el propio saber y una incesante búsqueda de lugar y mérito de la carrera, en una provincia sin tradición de formación de profesionales de este perfil y de un nuevo profesional en el campo de la educación cubana.

Constituyéndose un reto mayor para sus iniciadores, tanto profesores como estudiantes, se abre una nueva Facultad de Ciencias Sociales y Humanísticas, iniciando con un primer año de la carrera de Estudios Socioculturales. En la facultad, al no existir precedentes en la provincia ni egresados de la misma, los primeros profesores fueron un compendio de todas las aéreas de las ciencias sociales cubanas: sociólogos, filólogos, psicólogos y filósofos, todos imprimiéndole una marcada tendencia de su saber a la formación del currículo a sus primeros estudiantes.

Esto conllevó a una acentuada individualidad, pues cada profesor, muy experto en sus saberes y ciencias, le propiciaron a los primeros estudiantes una sensación de desánimo, dado por la poca fe en esta nueva formación. También las asignaturas se inclinaron por los saberes propios recibidos de sus carreras, tal es el caso de la cultura cubana, en la cual hubo una tendencia literaria en este sentido $y$ en otra un mayor impulso sociológico, lastrando la formación transdisciplinar de la que se hace gala con esta nueva área del saber, adoleciendo espacios de debate y búsqueda de consensos, por lo cual se evidenciaron deficiencias en el orden metodológico, estableciéndose un choque generacional en el trazado de líneas y modos de investigación, entre profesores de filosofía y egresados de la carrera.
Con el paso del tiempo se fue dando un perfeccionamiento de la carrera a nivel nacional y en la preparación de los profesores, se realizaron sesiones científicas dándose un creciente interés, lo cual fue imprimiendo un sentimiento de mayor identificación con la formación de este profesional y se ha logrado un claustro en la mayoría de egresados, que se especializan en las asignaturas y que se matriculan en cursos de superación científica y académica.

Sin olvidar un dolor latente en los egresados que sufren manifestaciones de desprecio de otros graduados, tanto de ciencias sociales y humanísticas como técnicas, en el caso de las primeras, se tilda esta carrera como última opción de estudio a estudiantes que se motivan por las ciencias sociales $y$ humanísticas $y$ en gran medida, por una débil orientación vocacional en este sentido, así como, una limitada gestión extensionista de la carrera, lo cual ha conllevado a un proceso de re-ubicación del egresado y un perfeccionamiento de las investigaciones. Actualmente, es una satisfacción mostrar mayor pertenencia y motivación de los estudiantes que ingresan, dado por la claridad y pasión que los profesores noveles le imprimen a la orientación vocacional cuando estos llegan al primer año.

Un fenómeno que ha incidido en la propagación de estudio, ha sido la apertura en las sedes universitaria municipales, lo cual hoy día se encuentra en un proceso de redimensionamiento pues no ha sido efectivo al no contarse con el máximo esfuerzo de muchos profesores que de forma adjunta colaboraron, así como de los estudiantes, que a diferencia del curso regular con sede en la universidad, no cuentan con una tradición de estudios que les permita la formación de las habilidades necesarias para su desarrollo profesional. Este planteamiento por si solo sería una investigación inacabada, dada las experiencias que en este orden se han logrado, pero se realiza el señalamiento para que se conozca de esta mayor apertura en el sistema educacional cubano.

Para finalizar y responder la pregunta que inicia este debate, la carrera puede $y$ debe dar un carácter privilegiado a la investigación 
sociocultural, como objeto de trabajo de este profesional, pues su primer reto esta en la comprensión de la realidad sociocultural, su decodificación y explicación - a partir de un proceso investigativo - para con ello, determinar los problemas de análisis y solucionarlos desde el trabajo social comunitario; ampliando además los instrumentales que en el orden metodológico se han venido acumulando en la práctica de desarrollo sociocultural comunitario del país, el subcontinente - América Latina posee una amplia $y$ rica experiencia $-y$ el mundo.

Es necesario un análisis más profundo y detallado de las demandas de este profesional en los diversos territorios en los que la carrera se ha implementado, debido a la implicación que el mismo tiene en el diseño de estrategias, proyectos y programas de desarrollo sociocultural a partir del estudio de las realidades socioculturales de las provincias, municipios y comunidades del país; la formación debe parecerse cada vez más a las necesidades concretas de los espacios territoriales.

Con el paso del tiempo de la carrera en la Universidad de Granma, para el año 2007, con la incorporación de profesores noveles, se creó el Departamento de Estudios Socioculturales que hasta el momento se nombraba Departamento de Humanidades. De esta manera, se separan los profesores recién llegados de estudios socioculturales de las demás áreas, aún cuando en el claustro se cuenta con sociólogos y filólogos. Es un colectivo de profesores muy jóvenes pero con gran interés de hacer $y$ de lograr mayor reconocimiento desde lo curricular y en el plano de la investigación, lo cual se avala con los resultados que se han obtenido en materia de investigación y con los resultados de fuertes impactos para el desarrollo del territorio.

\section{ENTONCES ¿DESDE DONDE NOS HABLAN LOS ESTUDIOS SOCIOCULTURALES?}

Para dar una debida respuesta, resulta importante aclarar que las experiencias que se someterán a juicios críticos de los lectores, se constituyen en este campo de investigación, en la Universidad de Granma.
Iniciando este acercamiento, se puede exponer que existen dos líneas de investigación fundamentales: la primera, denominada trabajo sociocultural comunitario y la segunda, estudios culturológicos. En ambas existen una serie de subtemáticas de interés investigativo para el territorio, tal es el caso de la: Implementación de estrategias y proyectos socioculturales encaminados a elevar la calidad de vida de la población. Estudio sobre la efectividad y proyección social de los proyectos de desarrollo comunitario en la provincia Granma. Pensamiento y política cultural: gestión e implementación, programación, animación sociocultural y promoción cultural. El trabajo sociocultural como ámbito de actuación profesional. El dilema ético de los estudios socioculturales. El componente cultural del desarrollo local en comunidades rurales de la provincia Granma. Estudios comparados: comunidades urbanas-comunidades rurales; comunidades rurales-comunidades rurales; comunidades urbanas-comunidades urbanas. Medio ambiente y desarrollo comunitario ${ }^{7}$.

Entre otras subtemáticas se encuentran: Expresiones y manifestaciones de la cultura popular tradicional en el ámbito sociocultural. Los procesos de construcción de la identidad cultural. Sus nexos con la historia y la cultura. Rol de las instituciones (culturales, educacionales, organizaciones políticas y de masas) y agentes socioculturales (comunidades, artistas, creadores e intelectuales). El patrimonio cultural, su rescate y conservación. Estudio y preservación del patrimonio cultural tangible e intangible. El patrimonio inmaterial de la provincia Granma. Fiestas populares y oralidad. Las artes plásticas en Granma. Memorias y contemporaneidad. Investigaciones sobre la religiosidad de Granma, aspectos socioculturales. Estudio de público, recepción y consumo cultural. Legitimación del producto artístico y literario. Acercamiento antropológico de los elementos identitarios y tradiciones de Granma. Ética profesional en la formación

$7 \quad$ Tomado de las líneas de investigación de Estudios Socioculturales, Facultad de Ciencias Sociales y Humanísticas, Universidad de Granma. Colectivo de autores. 
del licenciado en Estudios Socioculturales. El pensamiento filosófico cubano. Su vigencia en la cultura cubana: Varela, Céspedes, Maceo, Martí, Mella, Villena, Enrique José Varona, Ernesto Guevara, Fidel Castro, entre otros ${ }^{8}$.

En las anteriores, entre otras, se constituyen algunas líneas de la investigación sociocultural, que dan énfasis a un análisis más detallado, si se profundiza en explicitar la dicotomía condicionadora de los procesos socioculturales, es decir, cómo son condicionados por procesos sociales, económicos, políticos, medioambientales, éticos y epistemológicos y cómo a su vez actúan como condicionadores de dichos procesos, para con ello entender las transversalizaciones de los Estudios Socioculturales. En términos metodológicos, se trata de tomar elementos específicos de un "escenario con una convocatoria instrumental amplia", donde confluyen enfoques disciplinarios diversos. Por supuesto, la presencia de esta multiplicidad de ejes obliga a la utilización de herramientas metodológicas de todas las ciencias explicativas de la Cultura.

Las herramientas metodológicas que tipifican un diseño de investigación y definen la investigación sociocultural, se permean de los métodos que le son comunes a las demás ciencias de la cultura, es decir, métodos y técnicas de la investigación social, tales como: la utilización de los métodos teóricos de la filosofía, así como, métodos cualitativos partiendo desde estudios fenomenológicos, etnográficos, etc. $y$ por supuesto, ante el predominio de lo cuantitativo en las ciencias sociales, métodos de este orden. Aunque dado el carácter transdisciplinar, se potencia en estas investigaciones, si bien lo cualitativo, también la utilización de ambos paradigmas, tomando el nombre de investigación sociocultural, convirtiéndose en

8 Tomado de las líneas de investigación de Estudios Socioculturales, Facultad de Ciencias Sociales y Humanísticas, Universidad de Granma. Colectivo de autores.

$9 \quad$ Hernández, Isabel. "Investigación sociocultural en población. Criterios estratégicos para América Latina y el Caribe". Serie: Población y Desarrollo. Investigación sociocultural. Documento nro.1. Santiago de Chile, 1994: 3. un espacio teórico metodológico y práctico que se va más allá de la explicación desde una sola ciencia de análisis de la realidad.

La transdisciplina se constituye como un enfoque novedoso, que lamentablemente aún no se ha permeado lo que debiera en nuestra práctica social, la cual lleva implícito un intercambio respetuoso de saberes, en el que ninguna de las disciplinas puede ser vista como "ciencia auxiliar", sino que el propósito de indagación es el que determina la prevalencia o no de las especificidades de cada saber. Para ello deben ser re-construidas las nociones desde una unificación conceptual co-participativa y sistémica.

Los Estudios Socioculturales tienen en la transdisciplina su distinción fundamental, aunque no es exclusiva de esta área de saber, le pertenece por la complejidad de su objeto de estudio. De esta manera, la explicación de lo que abordan parte del supuesto de que la realidad sociocultural constituye un todo complejo y sistémico, mediado por interrelaciones de tipo social, cultural, económicas, medioambientales, etc. $y$ que esta es el objeto desde donde nos hablan los estudios socioculturales. Parecería que habría que reinventar la realidad, al punto de reconstruirla con base en los cambios de términos, pero sencillamente el discurso desde los estudios socioculturales no es una visión lineal sino un proceso de estudio de la realidad a través de la explicación y comprensión de los problemas socioculturales.

La carrera ha realizado excelentes investigaciones como parte de la culminación de estudios de los estudiantes con desempeño investigativo en sus cinco años, logrando un marcado impacto en el territorio por los aportes teóricos y prácticos que obtienen mediante el proceso de presentación y aprobación de los resultados.

Se deben analizar también las deficiencias para ver desde donde estas nos han hablado $y$ a qué se le ha echo caso omiso. Además, se debe mencionar el daño, por decirlo de alguna manera, de la tendencia al sociologicismo, pues en la formación de este profesional existe una inclinación hacia esta rama dejando atrás otro tipo de estudios. Se consideran problemas $y / u$ 
objetos de este tipo de investigación: la carencia en algunos momentos de suficiente conocimiento sobre la pertinencia de temas de interés cultural, por ejemplo: tatuajes, moda, identidad juvenil, ente otros, lo cual evidencia que ha existido un desequilibrio por la influencia de los temas del tratamiento comunitario y problemas de otra índole, siempre permeados por estereotipos que han precedido en los estudios culturales y sociales.

Muchas han sido las limitaciones en el espacio de formación, pero los logros están yendo mas allá del permeado positivismo en los cientistas sociales $y$ del poco estudio que muchos le han dedicado a este tema, pues cabe decir que en su gran mayoría se han hecho eco de lo que otros han dicho, para lastrar los resultados, pero sin lugar a dudas el proceso de construcción de este profesional va más allá de incomprensiones y desánimos, dado lo meritorio de los resultados que se han alcanzado $y$ en las actuales proyecciones de desarrollo en la carrera.

La realidad de hoy apunta a que la necesidad de este tipo de estudios, amerita un basamento diferente con re conceptualizaciones sobre la base de la experiencia cubana y sobretodo con una apertura hacia nuevos retos en las ciencias sociales cubanas, siendo una oportunidad única de realizar practicas de autorreconocimiento desde nuestra propia realidad, la cual es en definitiva, el lugar desde donde nos hablan los estudios socioculturales.

\section{CONCLUSIONES}

El sistema de educación superior en Cuba y las ciencias sociales en general, han sido protagonistas de la apertura de la carrera de Estudios Socioculturales, siendo un reto en el ámbito cultural cubano.

A esta carrera le es inherente la transdisciplina como herramienta metodológica, por su utilidad para el abordaje desde disciplinas diversas que confluyen respetuosamente en el análisis de una realidad compleja.

Aún cuando su desarrollo en Cuba es incipiente, la reformulación de la carrera de Estudios Socioculturales, en función de un perfeccionamiento del Plan de Estudios y de un re-diseño de sus esferas y campos de actuación, contribuirá a un acercamiento mucho más profundo a la realidad sociocultural del país y con ello al diseño de estrategias, proyectos y programas de desarrollo sociocultural mucho más coherentes con las demandas del contexto local, nacional y mundial en el que estamos insertos.

\section{BIBLIOGRAFÍA}

Barnet, Miguel. "La razón del otro: ¿para qué sirve la Antropología hoy?". Revista Cubana de Antropología 3 (5). 2002: 6-12.

Basail, Alain. "Un acercamiento al objeto de estudio de la Antropología". Revista Cubana de Antropología 3 (5). 2002: 17-20.

Colectivo de autores. Modelo del profesional de la especialidad en Estudios Socioculturales. Ministerio de Educación Superior en Cuba.

Colectivo de autores. Plan de estudios de la carrera de Estudios Socioculturales. Ministerio de Educación Superior en Cuba.

Colectivo de Autores. Modernidad. Posmodernidad. Colección Pensar en Cuba. La Habana: Editorial de Ciencias Sociales, 1998.

Follari, Roberto. "Estudios sobre postmodernidad y Estudios Culturales ¿sinónimos?”. Revista Latina de Comunicación Social 35. 2000. En:

<http://www.ull.es/publicaciones/latina/ argentina2000/15follari.htm>

González Rey, Fernando. Problemas epistemológicos de la Psicología. La Habana: Editorial Academia, 1996.

Guanche, Jesús. "Antropología y transdisciplinariedad". Revista Cubana de Antropología 3 (5). 2002: 13-16.

Hernández, Isabel. Investigación Sociocultural en población. Criterios estratégicos para América Latina y el Caribe. Chile: EATFNUAP/FLACSO, 1994.

Kleymeyer, Charles D. (comp.). La expresión cultural y el desarrollo de base. Quito, Ecuador: Ediciones ABYA YALA, 1994. 
Marcarian, E. S. "Lugar y papel de las investigaciones de la Cultura en las ciencias sociales modernas". J. Dumoulin (comp.). Cultura, sociedad y desarrollo. La Habana: Editorial Ciencias Sociales, 1973.

Monal, Isabel. "La Antropología y la Filosofía". Revista Cubana de Antropología 3 (5). 2002: 21-23.

Pérez de Cuellar, Javier (dir.). "Nuestra diversidad creativa". [Informe de la Comisión Mundial Cultura y Desarrollo]. México: Ediciones UnESCO, 1997.

Rodríguez, Raúl. El significado en los objetos sociales. Argentina: Dirección General de
Publicaciones de la Universidad Nacional de Córdoba, 1996.

Rosabal Navarro, Yanet. Lo sociocultural o la recreación del discurso culturológico en los marcos del contexto científico y tecnológico postmoderno. Pág. 15.

Touraine, Alain. Las transformaciones sociales del siglo $X X$. En: <http://inicia.es/de/ cgarciam/touraine01.htm>

Fecha de ingreso: 30/04/2011 Fecha de aprobación: 07/11/2011 